\title{
Polarized neutron spectrometer for inelastic experiments at J-PARC
}

\section{Status of POLANO Project}

Tetsuya Yokoo ${ }^{1,2, a}$, Kenji Ohoyama ${ }^{3,4}$, Shinichi Itoh ${ }^{1,2}$, Kazuaki Iwasa ${ }^{5}$, Naokatsu Kaneko ${ }^{1,2}$, Junichi Suzuki ${ }^{6}$, Manabu Ohkawara ${ }^{3}$, Kazuya Aizawa ${ }^{2}$, Seiji Tasaki ${ }^{7}$, Takashi Ino ${ }^{1,2}$, Kaoru Taketani ${ }^{1,2}$, Shigeru Ishimoto ${ }^{1}$, Masayasu Takeda ${ }^{8}$, Takayuki Oku ${ }^{2}$, Hiroshi Kira ${ }^{9}$, Kouichi Hayashi ${ }^{3}$, Hiroyuki Kimura ${ }^{10}$ and Taku J. Sato ${ }^{10}$

1 IMSS, High Energy Accelerator Research Organization, Tsukuba, Ibaraki 305-0801, Japan

2 J-PARC Center, Tokai, Ibaraki 319-1195, Japan

3 IMR, Tohoku University, Sendai, Miyagi 980-8577, Japan

4 WPI-AIMR, Tohoku University, Sendai, Miyagi 980-8577, Japan

5 Department of Physics, Tohoku University, Sendai, Miyagi 980-8578, Japan

6 MEC, High Energy Accelerator Research Organization, Tsukuba, Ibaraki 305-0801, Japan

7 Department of Engineering, Kyoto University, Kyoto 606-8501, Japan

8 JAEA, Tokai, Ibaraki 319-1184, Japan

9 CROSS,Tokai, Ibaraki 319-1106, Japan

10 IMRAM, Tohoku University, Sendai, Miyagi 980-8577, Japan

\begin{abstract}
Construction of the newly developed polarization analysis neutron chopper spectrometer (POLANO) commenced in the Japan Proton Accelerator Research Complex (J-PARC), Materials and Life Science Experimental Facility (MLF). The POLANO is a direct geometry chopper spectrometer with neutron polarization analysis capability. In the suite of inelastic spectrometers, six instruments are now in operation. POLANO will be the only spectrometer dedicated to polarization analysis experiments. The primary phase of the construction will be completed by 2014 with beam commissioning scheduled for 2015.
\end{abstract}

\section{Introduction}

Although the polarized neutron technique has been developed and used for many years, the application of the time-of-flight (TOF) method has only been realized in recent years. In particular, with regard to the inelastic spectrometer, the polarized neutron technique finds limited practical use in wide scattering angle instruments. In the light of recent discoveries in material science, many of the observed complex phenomena are largely due to the entangled physical degrees of freedom of spins, charges, orbitals, and even lattice vibration. In neutron scattering experiments, the varying dependence of momentum, energy, and temperature can be interpreted by these of degrees of freedoms. However, a unique, effective, and direct way to observe these properties separately is via the polarization analysis. The polarization analysis neutron spectrometer POLANO enables polarization analysis of inelastic scattering experiments to investigate the dynamical properties of the above mentioned multiple degrees of freedom. POLANO is a collaborative project between KEK and Tohoku University under the aegis of KEK Inter-University Research Program. The project has been launched in 2009 starting as an S-type project with financial support from the KENS neutron facility.

a e-mail: tetsuya.yokoo@kek.jp
After the budget was successfully sanctioned and obtained for the project, actual construction was initiated in 2013. Although a part of the instrumental design is still under discussion, the main conceptual design and manufacturing of the main components are expected to be completed by the end of 2014. In the Materials and Life Science Experimental Facility (MLF) at the Japan Proton Accelerator Research Complex (J-PARC), there is a plan to apply the polarization technique to the instruments as small angle scattering, reflectometer, imaging instruments, and so on. For inelastic scattering, there will be six beam lines involving direct geometry, inverted geometry, and spin echo instruments. POLANO will be the direct geometry chopper spectrometer and the only instrument with polarization analysis capability.

\section{Shielding and beam transport}

The spectrometer concepts are compact design with reasonably wide sample space, higher neutron flux, higher neutron energy polarization up to $100 \mathrm{meV}$, and medium resolution. In order to achieve these concepts, geometrical conditions of spectrometer were chosen as follows, moderator-sample distance $L_{1}=17.5 \mathrm{~m}$, sample-detector distance $L_{2}=2.0 \mathrm{~m}$, and Fermi chopper-sample distance $L_{3}=1.85 \mathrm{~m}$. We used a slightly shorter distance in $L_{2}$

This is an Open Access article distributed under the terms of the Creative Commons Attribution License 4.0, which permits unrestricted use, distribution, and reproduction in any medium, provided the original work is properly cited. 


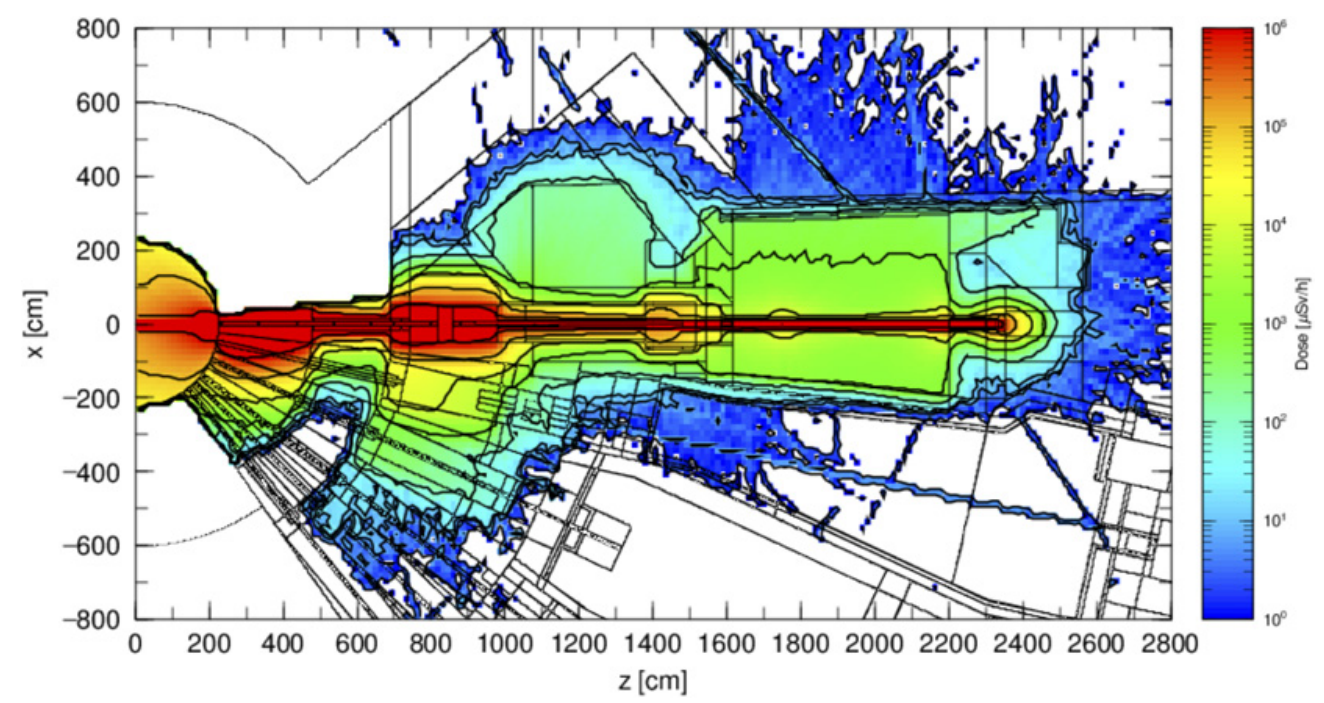

Figure 1. Typical calculated result of ray tracing Monte Carlo simulation. The sum of the $\gamma$-ray and neutron doses is mapped as a contour plot. The neutron source is beam line BL23 viewing decoupled moderator of the MLF.

from Ref. [1], but the energy and momentum resolution are not significantly affected.

The shielding design was evaluated based on $\gamma$ and neutron ray tracing by using the Monte Carlo PHITS code. Figure 1 shows an example of the calculated $\gamma$ and neutron dose distribution. The POLANO is viewing a decoupled moderator that is one of three types of hydrogen moderators (coupled, decoupled and poisoned), and the view of the moderator is $10 \mathrm{~cm}$ square of cross section. A radiation dosage of less than $6.25 \mu \mathrm{Sv} / h$ of surface dose was achieved with concrete, steel and boric acid as shielding materials. In certain parts of the shielding in the vicinity of the sample space, stainless steel was used as a non-magnetic material in order to make possible the conduction of high-magnetic field experiments.

To maximize the beam flux at the sample position, focusing supermirror guide tubes with $m=4$ have been installed as reported in Ref. [1,2]. The use of an elliptical guide tube provides a flux that is 10 times higher flux than no mirror guide tube (collimator) even for $100 \mathrm{meV}$ neutron (data are not shown here). For the monochromatic beam, we also evaluated the neutron flux at the sample position and the resolution of the diffracted beam at the detector position by using McStas [4,5], which information is shown in Fig. 2. Figure 2a depicts the flux observed in an area of $2 \times 2 \mathrm{~cm}^{2}$ after the beam passed through a Fermi chopper rotating at frequencies at 200, 400 and $600 \mathrm{~Hz}$. The dimensions of the Fermi chopper are rotor diameter $D=80 \mathrm{~mm}$, slit width $d=2 \mathrm{~mm}$, and curvature radius $r=450 \mathrm{~mm}$ with a transmission efficiency of 0.8. Since POLANO is aimed at the incident neutron energy range up to $E_{i}=100 \mathrm{meV}$, the evaluated intensity and resolution below $100 \mathrm{meV}$ are reasonable for the polarization analysis experiments. Further, to estimate the energy resolution, a vanadium cylinder with outer radius of $12.5 \mathrm{~mm}$ inner radius of $12 \mathrm{~mm}$ and height of $4 \mathrm{~cm}$ was set at the sample position. An energy resolution of $4 \sim 5 \%$ can be obtained below $100 \mathrm{meV}$ with the integrated intensity over $1 \times 10^{5}\left[1 / \mathrm{s} / \mathrm{cm}^{2}\right]$ at the sample position for an accelerator power of $1 \mathrm{MW}$ as shown in Fig. $2 \mathrm{~b}$.

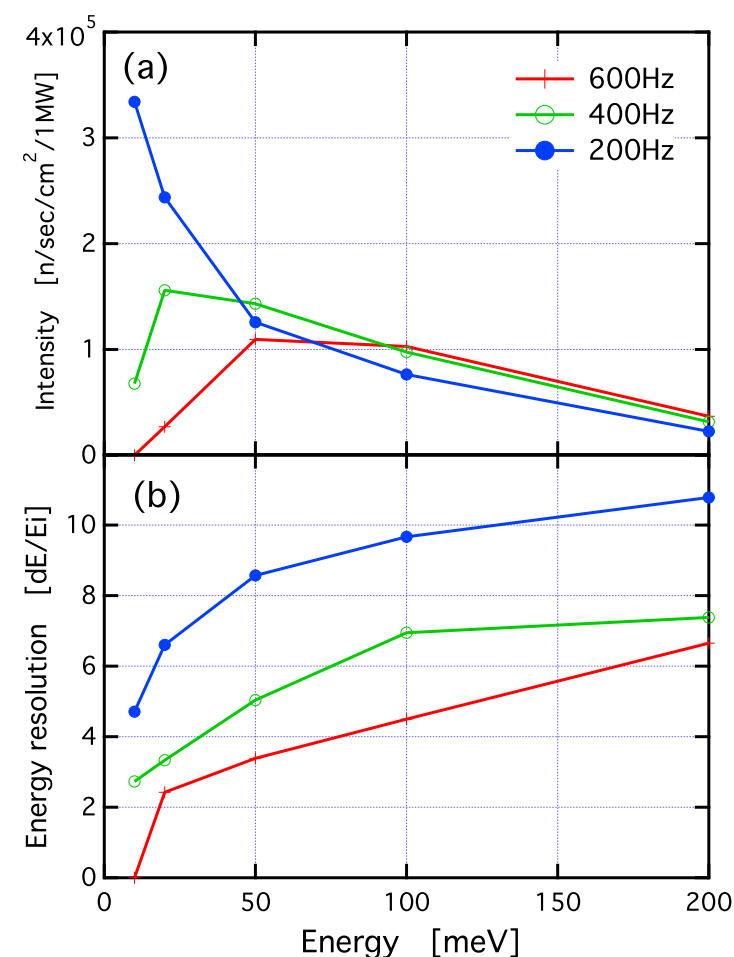

Figure 2. (a) Neutron intensity and (b) energy resolution estimation by McStas. monochromatized beam obtained by a Fermi chopper rotating at 200,400, $600 \mathrm{~Hz}$ is used.

Since the focusing guide tubes affect beam divergence, we estimate the beam profile as well. Figure 3 shows the intensity distribution at $L=16.435 \mathrm{~m}$ from the moderator, where a SEOP ${ }^{3} \mathrm{He}$ filter cell will be placed. The beam width at the ${ }^{3} \mathrm{He}$ filter has a full width of $8 \mathrm{~cm}$ at the bottom, and $4 \mathrm{~cm}$ at the half height. In the early stage, ${ }^{3} \mathrm{He}$ cells with a diameter of $5 \mathrm{~cm}$ will be used because the ${ }^{3} \mathrm{He}$ polarizing technique has already been established for $5 \mathrm{~cm}$ cells. Since cells with a diameter of $10 \mathrm{~cm}$ have now been developed, the beam exiting the polarizer will be almost fully polarized in the final stage. 

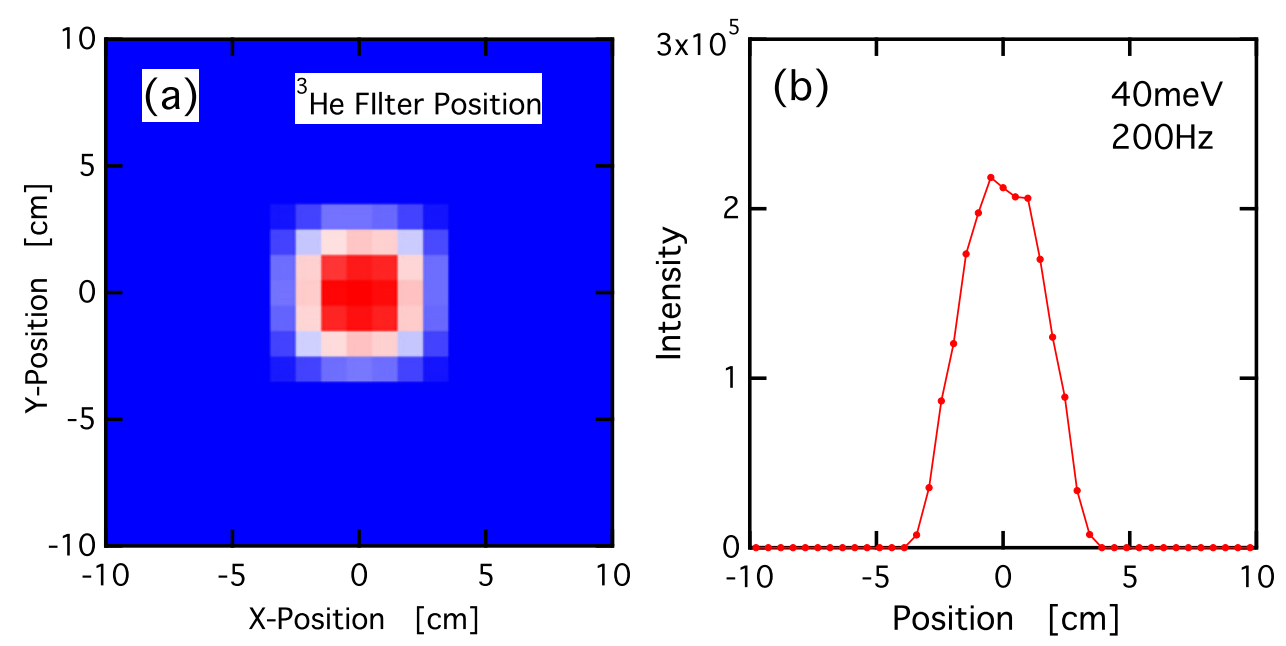

Figure 3. (a) Neutron intensity distribution at the ${ }^{3} \mathrm{He}$ cell position. (b) $1 \mathrm{D}$ plot at $\mathrm{Y}=0 \mathrm{~cm}$.

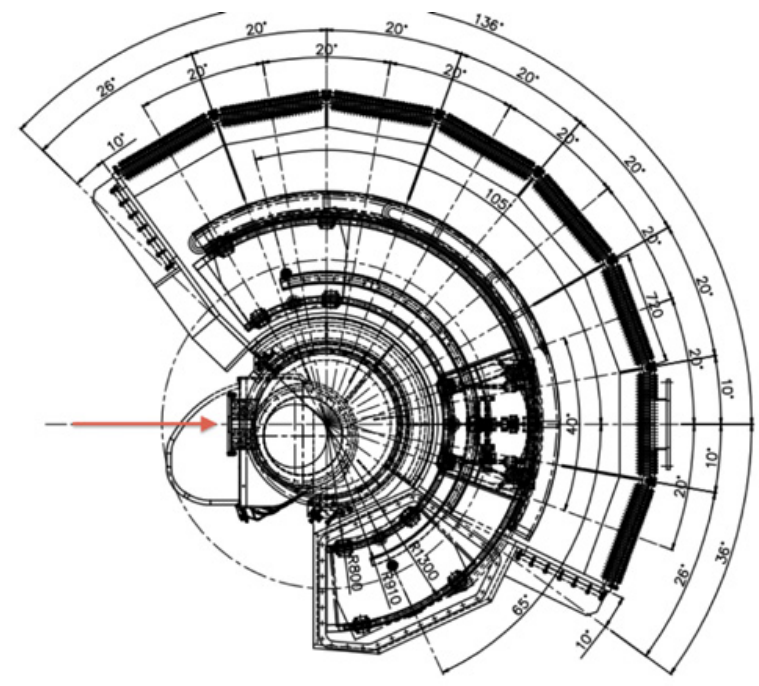

Figure 4. Top view of planned vacuum chamber. The neutron beam direction is indicated by the red arrow.

\section{Vacuum chamber and detectors}

A vacuum chamber is to be installed at the sampledetector section to avoid air scattering and to ensure evacuated insulation for the refrigerator. Figure 4 shows the current design of the vacuum chamber. To ensure flexible use of the sample space, the chamber is designed to be detachable, and it is composed of three parts. The sample chamber is the first chamber, in which the sample is placed and the sample environments are set. The second is a connecting chamber that connects the sample chamber and scattering chamber. Both the sample and scattering chambers are sealed by thin $(t=1.5 \mathrm{~mm})$ aluminum windows, and the chambers are completely isolated from air. Certain magnetic devices such as the spin flipper (positioned after the sample) will be installed in this section. The third is the scattering chamber wherein the suite of analyzer mirror and detectors are placed. In addition, $\mathrm{B}_{4} \mathrm{C}$ vanes and liners are planned to be installed in the chamber [3].
At the end of the scattering chamber, position sensitive detectors (PSDs) are to be installed on the inside of the chamber. For energy range of the POLANO, large pressure of ${ }^{3} \mathrm{He}$ gas is not required to achieve reasonable detector efficiency. Therefore, we adopted the use of a $10 \mathrm{~atm}$ gas pressure PSD with $600 \mathrm{~mm}$ effective length and $3 / 4^{\prime \prime}$ diameter. An efficiency of about $70 \%$ is expected for $100 \mathrm{meV}$ neutrons. The PSDs are arranged in three layers in the vacuum chamber. The horizontal scattering angle (detector angle) ranges from $-30^{\circ}$ to $130^{\circ}$, and the range is $\pm 25^{\circ}$ along the vertical direction. At first, only horizontal (center) layers are to be fully installed for polarization analysis experiments.

\section{Polarizer and analyzer}

The polarizer, analyzer, and other magnetic devices installed in the POLANO are currently under development $[6,7]$. As the polarizer, a cylindrical ${ }^{3} \mathrm{He}$ spin filter (HeSF) based on the spin-exchange optical pumping (SEOP) technique will be used to polarize neutrons in the wide energy range. During the experiments, the $\mathrm{HeSF}$ is operated in the on-beam mode to maintain ${ }^{3} \mathrm{He}$ polarization. A HeSF with a diameter of $3 \mathrm{~cm}$ will be installed in the POLANO in the spring of 2015. HeSF systems with larger diameters $(5$ to $7 \mathrm{~cm}$ ) are now under development. We have already succeeded in preparing a GE180 cell with a diameter of $10 \mathrm{~cm}$.

One of the serious technical issues affecting POLANO is the choice of a spin analyzer with a large solid angle. Since the technology concerning polarized ${ }^{3} \mathrm{He}$ in GE180 glass cells with large solid angles is not currently available in Japan, a phased approach needs to be adopted for the spin analyzer to be used in POLANO. In the first stage, a $5.5 Q_{c}$ supermirror bender analyzer with a covered scattering angle of about $18^{\circ}$ will be installed on a rotatable stand around the sample because it is most practical in the thermal neutron range. The distance between the sample and the aperture of the supermirror will be $850 \mathrm{~mm}$. The $5.5 Q_{c}$ supermirror can cover up to $E_{f} \sim 40 \mathrm{meV}$, corresponding to an energy excitation range of 50 to $60 \mathrm{meV}$. This energy range is of great 


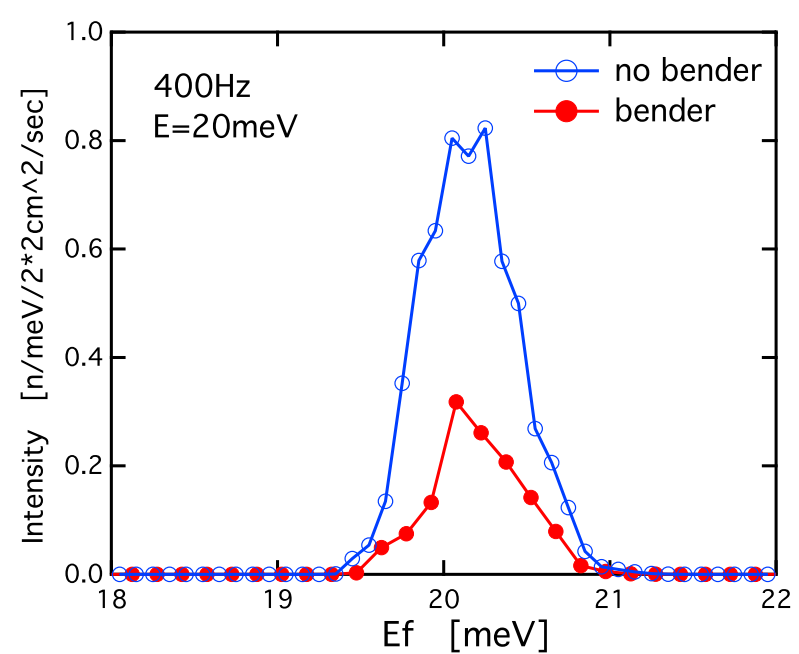

Figure 5. Estimation of transmission of a supermirror bender for $E=20 \mathrm{meV}$ by McStas. Red and blue lines indicate the transmitted neutron intensity from a vanadium rod with and without the bender, respectively.

scientific interest, corresponding to phenomena such as spin-lattice couplings in multiferroic systems, geometrical spin frustration systems, and multipole orderings in rare earth compounds. On the other hand, since we have installed a focusing guide tube between the moderator and the Fermi chopper to obtain high flux at the sample position, it is necessary to ensure matching of the angular divergence of the focusing guide tubes and analyzer.

Figure 5 shows the results of the estimated intensity from a vanadium rod at the sample position observed by means of a POLANO detector for $E=20 \mathrm{meV}$, which were calculated with McStas. The transmission of the expected $5.5 Q_{c}$ supermirror bender is obtained as approximately 0.33 for $20 \mathrm{meV}$, which is close to the ideal value of 0.35 , thereby indicating that the matching of the analyzer with the incident beam divergence is satisfactory. In the second stage, a banana-shaped HeSF will be installed as a spin analyzer to study neutrons in the $100 \mathrm{meV}$ region. The large solid angle HeSF will be installed at a position close to the sample and will be used in the off-laser mode; consequently, the polarization will undergo decay. The technology to polarize ${ }^{3} \mathrm{He}$ in a banana-shaped GE180 cell and achieve homogeneous magnetic fields for the analyzer should be simultaneously developed in the first stage. PASTIS type or vertical solenoid type coils are expected to be used for the generation of magnetic fields for the analyzer.

\section{Chopper devices}

One of the most essential devices for this type of instrument is the chopper. We have developed several types of choppers originally for the high resolution chopper spectrometer HRC in the MLF [8,9]. POLANO will basically use T0 and Fermi choppers with the same specifications as those of the HRC. In addition, we plan to use slow rotating disk choppers (band chopper) and a fast rotating disk chopper (correlation chopper) to achieve cross-correlation. The T0 chopper is known as a device

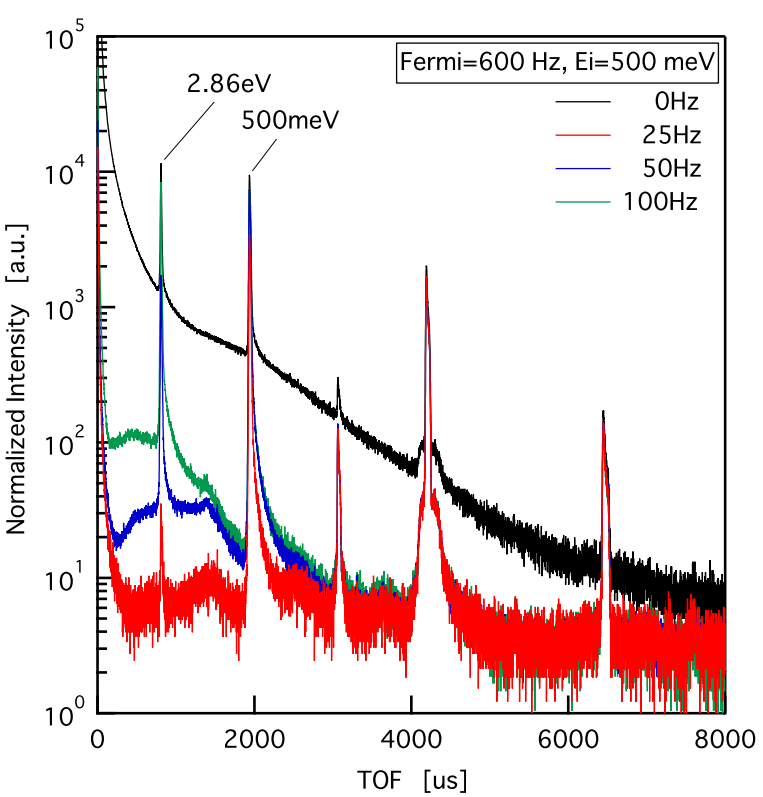

Figure 6. Suppression of background using newly developed T0 chopper at various rotational frequencies. The spectra are monochromatized by the Fermi chopper.

that mechanically reduces the intensity of prompt $\gamma$ rays and fast neutrons. For POLANO a T0 chopper with a frequency of $f=100 \mathrm{~Hz}$ of Inconel X750 blade is adopted for the parameters of beam window width of $w=80 \mathrm{~mm}$, width of the blade $w+2 \Delta w=82 \mathrm{~mm}$, length of the blade (along neutron beam direction) $l=300 \mathrm{~mm}$, and radius of the blade $R=300 \mathrm{~mm}$. The time of the blade to be removed from the beam cross section is therefore easily evaluated as $t_{0}=(w+\Delta w) /(2 \pi R f)$. Since the blade margin $\Delta w$ corresponds to a type of rotational jitter, the phase control accuracy should be less than $\pm 5 \mu$ s with respect to the timing of the neutron pulse production. Figure 6 shows the performance of the developed T0 chopper when the neutron beam is operational (from Ref. [8] Fig. 11). In this test experiment, the incoming neutron beam is monochromatized by a Fermi chopper optimized at $E_{i}=500 \mathrm{meV}$. The several other observed peaks are due to the multi- $E_{i}$ process, and we observe higher energy neutrons even at $2.9 \mathrm{eV}$. At $500 \mathrm{meV}$ of energy $(2000 \mu \mathrm{s}$ of TOF) the background is reduced by nearly $1 / 100$ that of the incoming beam, and thus the chopper can be considered as exhibiting a satisfactory performance.

In Fig. 7, the photograph of a manufactured Fermi chopper that is similar type to the one developed for the HRC is shown. The following are the design parameters: slit width $w=1.2 \mathrm{~mm}$, slot (absorber) width $d=0.3 \mathrm{~mm}$, and rotor diameter $D=98 \mathrm{~mm}$. A boron-epoxy composite is used as neutron absorbers in the slit package of the rotor [9]. The maximum rotational frequency $f_{\max }=$ $600 \mathrm{~Hz}$ is controlled by $25 \mathrm{~Hz}$ step as tunes with proton pulse repetition. The phase can be controlled fairly precisely (see Ref. [9]); we achieved a phase accuracy of less than $0.3 \mu \mathrm{s}$ at $600 \mathrm{~Hz}$. The Fermi chopper is controlled by an auto exchanger, which enables switching between the Fermi chopper and guide tube by remote control. 


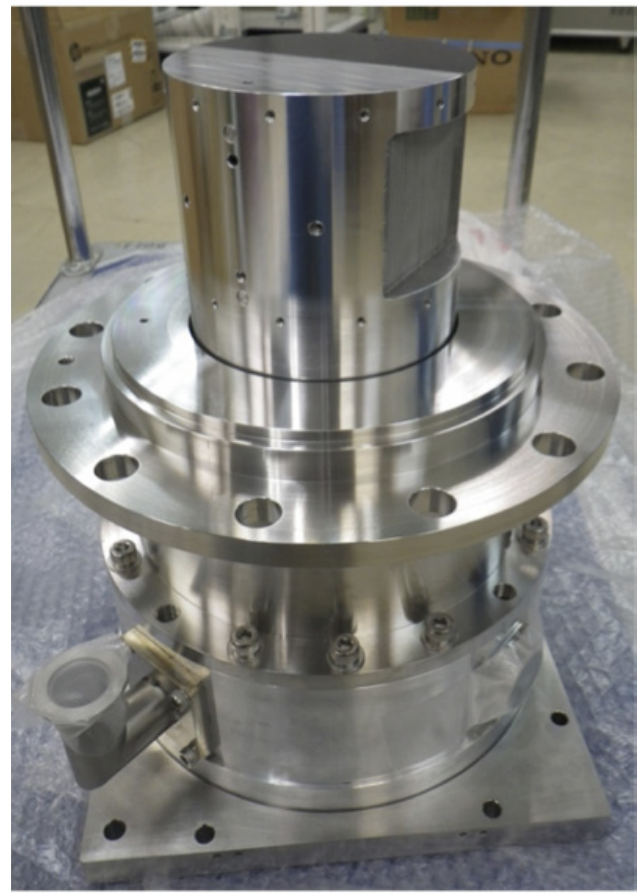

Figure 7. Newly assembled Fermi chopper.

Two band choppers will be placed along the beamline to avoid frame overlap. This type of chopper is a single disk chopper made of aluminum coated with boron enriched $\mathrm{B}_{4} \mathrm{C}$ with epoxy resin. Two disks rotate at $25 \mathrm{~Hz}$ with different opening angles to select the required channels. A fast rotating disk chopper is used to achieve cross correlation. The optimal parameters for the disk are a diameter range of 800 to $1000 \mathrm{~mm}$ with frequency of $350 \mathrm{~Hz}$, and 255 of open-close (slits and slots) sequence following the maximum length shift register sequence. According to the analysis of the disk via the finite element method (FEM), nearly $4000 \mathrm{MPa}$ of stress is applied at the end of the disk. This force can easily destroy metals. The correlation chopper is currently being developed with a slightly downsized disk.

\section{Summary}

In summary, the construction of the POLANO spectrometer for neutron polarization analysis has begun. The design of most instruments has been completed, and the main components are to be installed by the end of 2014 . R\&D of certain devices, particularly the polarization and magnetic devices still needs to be performed. In 2015, we plan to carry out radiological assessments. Neutron beams are expected to be operational for the POLANO in the beginning of 2015 .

The POLANO project was approved by the Neutron Scattering Program Advisory Committee of IMSS, KEK (Proposal No. 2009S09 and No. 2014S09). TY is partly supported by a JSPS KAKENHI Grant (Scientific Research (C) No. 26400376 and Scientific Research (B) No. 25287081). Further, TY and SI are supported by a JSPS KAKENHI Grant (Scientific Research (C) No. 24540352). OK was supported by a Grantin-Aid for Challenging Exploratory Research (24656004), Scientific Research (A)(23244068), (S)(23224009), MEXT, Japan.

\section{References}

[1] T. Yokoo et al., J. Phys. Soc. Jpn. 82, SA035 (2013)

[2] T. Yokoo et al., J. Phys. Conf. Series 502, 012046 (2014)

[3] T. Yokoo et al., Review of Scientific Instruments 82, 095109 (2011)

[4] K. Lefmann and K. Nielsen, Neutron News 10, 20 (1999)

[5] P. Willendrup, E. Farhi and K. Lefmann, Physica B 350, 735 (2004)

[6] K. Ohoyama et al., J. Phys. Soc. Jpn. 82, SA036 (2013)

[7] K. Ohoyama et al., J. Phys. Conf. Series 502, 012051 (2014)

[8] S. Itoh et al., Nucl. Instrum. Meth. A 661, 86 (2012)

[9] S. Itoh et al., Nucl. Instrum. Meth. A 661, 58 (2012) 